\title{
Application of automated systems for quality control of ground anti-icing treatment of aircraft
}

\author{
Oleg Dolgov ${ }^{1, *}$, Boris Safoklov ${ }^{1}$, Svetlana Sergeeva ${ }^{2}$, and Anna Ivanova ${ }^{2}$ \\ ${ }^{1}$ Moscow Aviation Institute (National Technical University), Volokolamckoe shosse, 4, Moscow, \\ 125993, Russia \\ ${ }^{2}$ Voronezh State Technical University, 20 years of October st.,84, Voronezh, 394006, Russia
}

\begin{abstract}
For the first time, this paper addresses the need to automate the maintenance of the quality control and control system at all stages and stages of the life cycle - anti-conduct fluid (POJ) for ground-based anti-dedealing treatment (POO) of an aircraft. The possibility of evolution of the quality control of the object of ground de-icing physical and chemical treatment of the surface of the aircraft - de-icing liquids, in "Management of quality - de-icing liquid as an object to ensure the safe take-off of the aircraft" is considered. The definition was introduced : "The quality of deicing liquid." To integrate into the system "Management of the quality of de-icing fluid" - on the basis of knowledge bases about THE POJ introduced as a functional concept "The quality of de-icing liquid at the POO stage". In order to introduce information software support for the POO process using databases and control systems, the concept of "Regeloscopic Ground De-Icing Protection Program of the Armed Forces" has been introduced, which will allow and move to an effective method of improving the quality of the service - integrated automation of the POO.
\end{abstract}

\section{Introduction}

Within the framework of flight safety there is a system of provisions, which interprets the inadmissibility of the aircraft's take-off if there are any contaminants on its carriers, control and other surfaces, including snow-ice deposits. This system of provisions provides for the complete cleaning of surfaces before take-off and control of the condition of the aircraft's surfaces in conditions of actual or predictable icing up to the executive launch and is called "the concept of a clean aircraft (aircraft)" [1]. Implementation of the concept terrestrial anti-pale treatment -POO (de-icing), groundde-icing protection - POS (antiicing), using de-icing liquids - POJ. The effectiveness of the process of removing snow-ice sediments from the surfaces of the aircraft in order to implement the concept of a "clean aircraft" depends, first of all, on the POJ itself, the effectiveness of the processes of preparation of the POJ for work, processes and operations on the ground POO (de-icing) with the formation of a protective layer, preventing re-ice formation on the processed surfaces of the aircraft (anti-icing) [2-3]. The processes of POOs (de-icing), PHO (antiantiicing), as providing the concept of a "clean aircraft" should create the conditions for the

\footnotetext{
*Corresponding author: $\underline{\text { dolgov@mai.ru }}$
} 
successful implementation of the main task - the safe take-off of the aircraft[1]. The material object by which mechanical, energy and physical-chemical processes on the treated surface of the POJ should be implemented and in which the technical and chemical processes should be implemented must meet the requirements, and to meet the established, current "necessity", as a result of providing consumers with the necessary set of properties of de-icing, POS (anti-icing) in specific consumption conditions.

To carry out the task of ensuring the safety of the aircraft's take-off, as a result of ground-based physical and chemical treatment, four types of liquid are used, classified by item I, II, III, IV, where I is Newtonian, II-IV non-Newtonian liquids. The rules of their production and their characteristics are set by standards - the expediency of the use of liquid type I - or liquids II-IV and regulated [2-3].

In order to prevent substandard ground-based service, the price of which - human lives carry out quality control measures of individual stages prior to the ground-based POA and directly in the process. It is excluded due to rationed indicators the use of substandard POJ works, the necessary quality control at the stages: procurement, transportation, reception, storage, as well as on the day of application and until the moment of application [4-5]. Given the wide geography of the use of PJ, the difference in weather conditions, the specifics of environmental safety standards and requirements for transportation, storage, use and operation of this type of liquids, as well as ensuring the cost-effectiveness of the process, the operator and the consumer, preparing the "De-icing Protection Of the Sun" "IOSA Standards Manual" is not enough to rely only on the narrow regulatory documentation, technical recommendations of the liquid manufacturer regulating the use of de-icing and anti-icing processing products, or the quality of production of a particular or the product[6-7]. Rules for the use of POJ should be formed not only on the basis of existing standards, recommendations and documentation from the liquid producer, but also on the basis of a proper comprehensive assessment of ice conditions, control of external meteorological conditions, control of changes in the concentration of the active substance and preparation of the relevant fluid prior to the process of de-icing and anti-icing.

For specific ground maintenance conditions, the Armed Forces define a set of basic functions of the POJ, i.e. properties that will ensure that the conditions for safe take-off are met within the concept of a clean aircraft [8-9]. The most difficult, at the same time, is the rapid response to the change in the conditions of use of liquid under a specific type of aircraft (aircraft) in specific meteorological (weather) conditions accompanying or prior to ice formation on the surface of the sun. In order for the POJ to perform the required functions during the processing process, it is necessary to carefully prepare the liquid: to select the type of liquid, based on the type of sun and weather conditions, to control the physical and chemical parameters of the solution, to regulate the concentrations of the active substance and components of its composition with the preservation of properties.

Given that the actual conditions of the POO (de-icing), the POS (anti-icing) of the Armed Forces are very different and can change over the course of the work, the POJ at this stage should be prepared for use with:

- saving processing time that prevents reprocessing when you take into account the originally incorrect conditions;

- saving the amount of solution used;

- saving the resources used to process machines;

- environmental safety of the use of solutions;

- cost-effectiveness.

From the foregoing we can see that only at the stage of terrestrial de-icing, from the entire spiral of the life cycle of the POJ, we are faced with the non-triviality of the task of quality control of the POJ, and this stage needs to be systematic and optimized [10-13]. 
If we take into account all the stages of the life cycle of the POJ, from the development and production process to the recycling process in terms of product quality management at all stages of the life cycle, then for operators (consumers) it is urgent and necessary to automate the process of quality management of the POJ, with the integration of this process into automated management systems of quality of ground airport services. There are no autonomous auto-laundered POO and business quality management processes to combat the ground icing of the armed forces [14-15].

\section{Experimental}

A. "The quality of de-icing liquid (POJ)."

As part of the automation of quality management, for promising functional models it is necessary to form a new concept for POJ as a material object and control object - "the quality of de-icing liquid" by giving it a definition:

"The quality of de-icing liquid (PIS) " is the ability to meet certain operational requirements, to meet international standards SAE AMS 1424 and ISO 11075:2007 (for type I liquids), SAE AMS 1428 and ISO 11078:2007 (for type II liquids, III, IV), to ensure the functional suitability, reliability of the removal of frozen precipitation and prevent their appearance on critical surfaces of the sun in order to ensure the safety of take-off, the absence of negative impact on the elements and paint coating of the sun, economy, environmental safety.

B. Control of quality - de-icing liquid

For the object of our interests - the POJ used in ground-based anti-pale-icing treatment POO (de-icing) As a natural evolutionary change, we propose to move from the current deicing - quality control of the entire phase of the product's life cycle to the quality management of the quality of the de-icing - de-icing fluid as an object of safe take-off of the aircraft, in the entire spiral of the product life cycle, from development to disposal.

Control of the quality of the PW for the use of liquid in the ground de-icing processing of the aircraft, and the preparation of the POJ for work - POO (de-icing), POS (anti-icing) of the aircraft (sun) as an independent process, as it exists today leads to an increase in the cost of processing. Arbitrary reduction of quality control points will result in a lack of a guaranteed result, and in turn, a possible increase in the amount of POJ expenditure at work, and then to increase the load and negative impact on the environment and economic losses. Accordingly, such approaches are not effective in today's environment.

The problem of optimizing the process of the regoloscopic program of ground de-icing protection of the armed forces needs to be solved. (regelo - ice removal, lat. the groundbased de-icing program of the aircraft).

For the model, let's define:

I - the process of THE POA is characterized by ambivalence, namely in the conduct of service work the task to provide the required level of quality, in our case it is the required level of security(S - safe) and with the increase of the $\mathrm{S} \rightarrow$ Smax should strive to reduce the economic costs (EE -expenses) of airlines $\mathrm{E} \rightarrow$ Emin.

II - in terms of model optimization - process efficiency as a result is determined by the Smax parameter.

To create a generalized model of the control system of this stage, you need to formalize the task.

Based on I accept that the task of optimizing the quality management of the regoscope program of ground de-icing protection of the sun is ambivalent (parameters $\mathrm{S}$ and $\mathrm{E}$ ).

The following main parameters are:

$\mathrm{S}$ - the quality of service in our case takeoff safety (Safe)

$\mathrm{T}$ - full process time (Time); 
R- resources needed to implement the process (Resourse);

In order to obtain a model, the regoscope program of ground de-icing protection of the armed forces must be linked to the parameters of the program described above (S, T, R).

Believing that the regoscope program of ground de-icing protection of the sun consists of n subprocesses [16].

Let's find that:

$s(t, r)$, for the i-process,

In the form of a work of features: $\mathrm{f}(\mathrm{t})$ and $\mathrm{k}(\mathrm{r})$

$$
\mathrm{S}_{\mathrm{i}}(\mathrm{t}, \mathrm{r})=\mathrm{P} \times \mathrm{f}(\mathrm{t}) \times \mathrm{k}(\mathrm{r}),
$$

when $i=1 \ldots \mathrm{n}, \mathrm{s}_{\mathrm{i}}-$ indicator of the quality of the $\mathrm{i}$-subprocess, $\mathrm{P}-$ conditions under which the process (normal multiplier) takes place, $t_{i}-i$-process time, $r_{i}-i$-process resources.

Take a nap:

$$
f_{i}(t)=F_{i}\left(1-e^{-a t_{i}}\right)
$$

$\mathrm{i}=1 \ldots \mathrm{n} ; \mathrm{F}_{\mathrm{i}}-$ limit $\mathrm{f}_{\mathrm{i}}(\mathrm{t})$ when $\mathrm{t} \rightarrow \infty$; $\mathrm{a}-$ POO time limit ratio, let's take for resources:

$$
\mathrm{k}_{\mathrm{i}}(\mathrm{r})=\mathrm{K}_{\mathrm{i}}\left(1-\mathrm{e}^{-\mathrm{bs}_{\mathrm{i}}}\right) \text {, }
$$

$\mathrm{i}=1 \ldots \mathrm{n} . \mathrm{K}_{\mathrm{i}}-$ limit $\mathrm{k}_{\mathrm{i}}(\mathrm{r})$ when $\mathrm{r} \rightarrow \infty ; \mathrm{b}$ - the cap-and-trade factor.

The generalized properties of the subprocesses of the entire regeloscopic program of ground de-icing protection of the aircraft form equality:

$$
\begin{aligned}
& \mathrm{S}=\sum \mathrm{s}_{\mathrm{i}}, \quad \mathrm{i}=1, \ldots, \mathrm{n} ; \\
& \mathrm{R}=\sum \mathrm{r}_{\mathrm{i}}, \quad \mathrm{i}=1, \ldots, \mathrm{n} ; \\
& \mathrm{T}=\sum \mathrm{t}_{\mathrm{i}}, \quad \mathrm{i}=1, \ldots, \mathrm{n} .
\end{aligned}
$$

From the above feature «Safe» i-process:

$$
\mathrm{s}_{\mathrm{i}}(\mathrm{t}, \mathrm{r})=\mathrm{P} \times \mathrm{F}_{\mathrm{i}}\left(1-\mathrm{e}^{-\mathrm{at}}\right) \times \mathrm{K}_{\mathrm{i}}\left(1-\mathrm{e}^{-\mathrm{bs}}\right), \mathrm{i}=1, \ldots, \mathrm{n} .
$$

According to the resulting system of equality, we have the following functional dependence:

$$
\mathrm{S}=\mathrm{P} \times \sum \mathrm{F}_{\mathrm{i}}\left(1-\mathrm{e}^{-\mathrm{at} \mathrm{t}_{\mathrm{i}}}\right) \times \mathrm{K}_{\mathrm{i}}\left(1-\mathrm{e}^{-\mathrm{bs_{ \textrm {i } }}}\right), \mathrm{i}=1, \ldots, \mathrm{n} .
$$

When $\mathrm{t} \rightarrow \infty$ and $\mathrm{r} \rightarrow \infty$,

$$
\mathrm{S}_{\max }=\mathrm{P} \times \sum \mathrm{F}_{\mathrm{i}} \times \mathrm{K}_{\mathrm{i}}, \mathrm{i}=1, \ldots, \mathrm{n} .
$$

$\mathrm{F}_{\mathrm{i}}=\mathrm{K}_{\mathrm{i}}=1, \mathrm{jr} \mathrm{S}_{\max }=\mathrm{P} \times \mathrm{n}$, therefore, $\mathrm{P}=\mathrm{S}_{\max } / \mathrm{n}$.

Accepting that the ultimate value of service quality $S_{\max }=1$.

Get:

$$
\mathrm{P}=1 / \mathrm{n}
$$




\section{Evaluation}

The resulting function of the regoscope program reflects the connection of the main parameters:

$$
\mathrm{S}=\frac{1}{\mathrm{n}} \times \mathrm{P} \times \sum \mathrm{F}_{\mathrm{i}}\left(1-\mathrm{e}^{-\mathrm{at}_{\mathrm{i}}}\right) \times \mathrm{K}_{\mathrm{i}}\left(1-\mathrm{e}^{-\mathrm{bs}}\right), \mathrm{i}=1, \ldots, \mathrm{n} .
$$

The resulting equality satisfies the set-in charge of Task II and allows for the further optimization of $\mathrm{E}_{\min }$ without violating this restriction by applying the following management strategies:

- In the conditions of minimal temporary $T_{\min }$ and resource $R_{\min }$ costs get the highest quality service $S_{\max }$

- For the minimum time $T_{\min }$ receives the $S_{\max }$ service to provide a minimum expenditure of $\mathrm{R}_{\min }$ resources

- With a minimum expenditure of $\mathrm{R}_{\min }$ resources on $\mathrm{S}_{\max }$ service to ensure the minimum time spent $T_{\min }$.

Any of the strategies presented takes into account the interests of all participants in the PROCESS (airport ground services), the airline, the requirements of the concept of "clean aircraft") $[17,18]$.

C. The quality of the POJ at the POO stage.

Forming the possibility of evolution, as the transition from "quality control" - stages to "quality management" throughout the spiral of the life cycle of the object of the POJ providing the implementation of the concept of "clean aircraft" and the introduction of automation in the process, we note that the set of expected operator (consumer) parameters of the quality of the de-icing liquid (POJ) (product) and their values, satisfying the needs, will be manifested in the process of de-icing. Let's highlight it in a separate stage (functional) of the life cycle spiral - "The quality of the POJ at the POO stage." For convenience, we will display the diagram of the blocks included in it in Figure 1 [19-20]. 


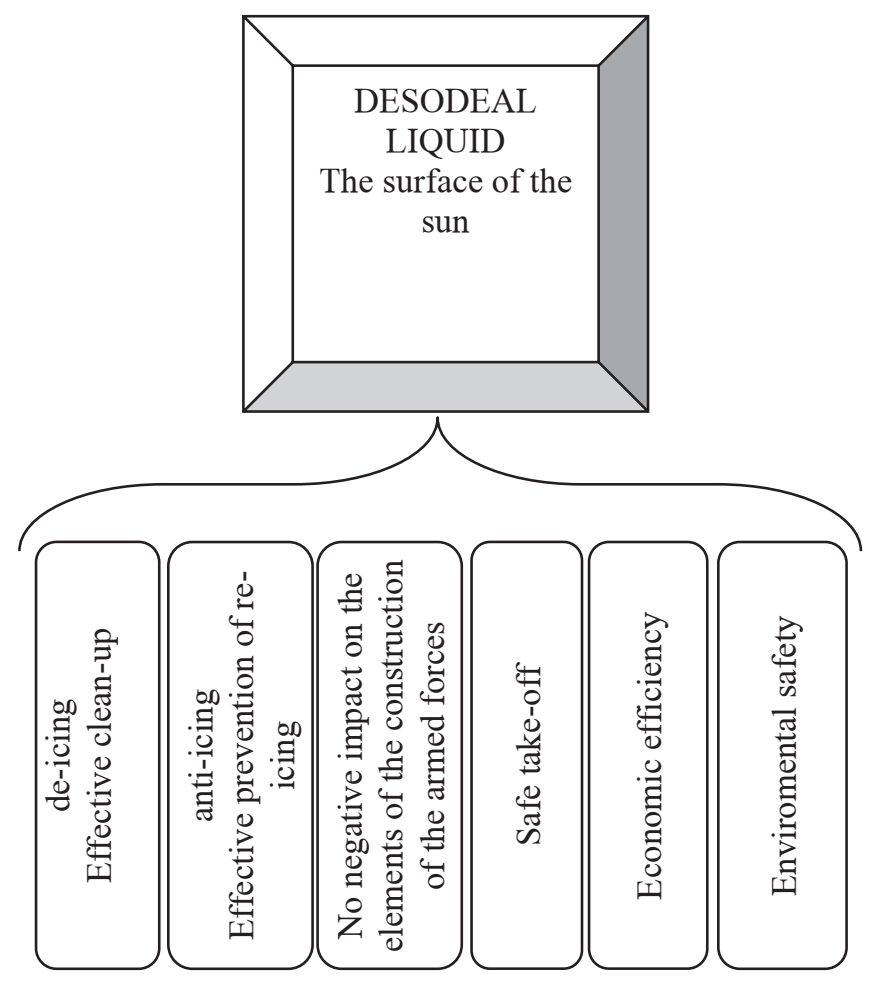

Fig. 1. Blocks included in the quality of the POJ at the POO stage

"The quality of the POJ at the POO stage" includes:

- lowering the freezing point of precipitation on the treated surface, washing the precipitation from the surface of the aircraft,

- formation of an external protective layer (ensuring that the surface of the aircraft is not icing again during the waiting period, while steering to the runway and running),

- no negative effect on the elements of the construction of the sun, paint coating, with the required level of the inhibitor in the liquid to protect against corrosion,

- ensuring the safe take-off of a "clean aircraft",

- estimated liquid flow rates (predicted sun processing time),

- no environmental burden on nature.

To integrate into the system "quality management - de-icing fluid" and build automation algorithms to the control object - "The quality of the POJ at the POO stage" is necessary:

- technologically sound restrictions on the properties of the POJ and the mode parameters of the POO technology. These restrictions may be supplemented or modified depending on specific conditions of application or new patterns are identified;

- classification of POJ and the method of selecting the type of POJ and optimal concentrations of active substances, ensuring maximum efficiency in the process of deicing, and as long as possible and fully preserving the properties of anti-icing, regardless of temperature, pressure, mechanical influences of the external environment and other factors characteristic of various meteorological, climatic, territorial conditions;

- mathematical models that describe the relationship between the composition of the formulation and the properties of the POJ; 
- the cost of consumables, as well as the standards of their consumption depending on the conditions of use and the type of liquid;

- the method of preparing a solution of the set (necessary in specific conditions) composition, and, therefore, the properties;

- the method of how to quickly change the required composition (and properties) depending on external factors;

- control and management of the environmental impacts of the use of POJ solutions.

- a criterion that allows you to choose the best version of the program from a variety of acceptable ones.

In turn, the main functions of "quality of life at the POJ stage" - we refer to the ability to:

- remove "ice" particles from the surface of the sun due to a mechanical "flush" with a chemical reduction of the freezing point of the solution compared to the freezing temperature of the pure solvent;

- washing mechanical particles from the surface of the sun and keeping them in a suspended state, and prevent deposition on the surface of the sun when processing is discontinued;

- ensure that the sun is effectively and completely cleaned of ice particles and mechanical particles, as well as take them out of the sun;

- provide quality protection to prevent ground icing;

- create favorable conditions for safe take-off;

- prevent negative impact on elements of the armed forces;

- be environmentally friendly,

- be resistant to other technical fluids accompanying the maintenance and operation of the sun, high temperature and pressure;

- have stable properties over time;

- to resist the transition of dissolved ice and impurities in it (dissolution, dispersing) into its composition.

D. "Regoloscopic program of ground de-icing protection of the armed forces"

As a universal criterion in the process of transition from "manual control" to process automation, with differentiated conditions of use of POJ, in the system "Management of quality - de-icing liquid"as an object to ensure the safe take-off of the sun, we introduce the concept - "Regeloscopic program of terrestrial de-icing protection of the sun" (regelo - ice removal, lat. ) presenting it as a scheme (Figure 2). 


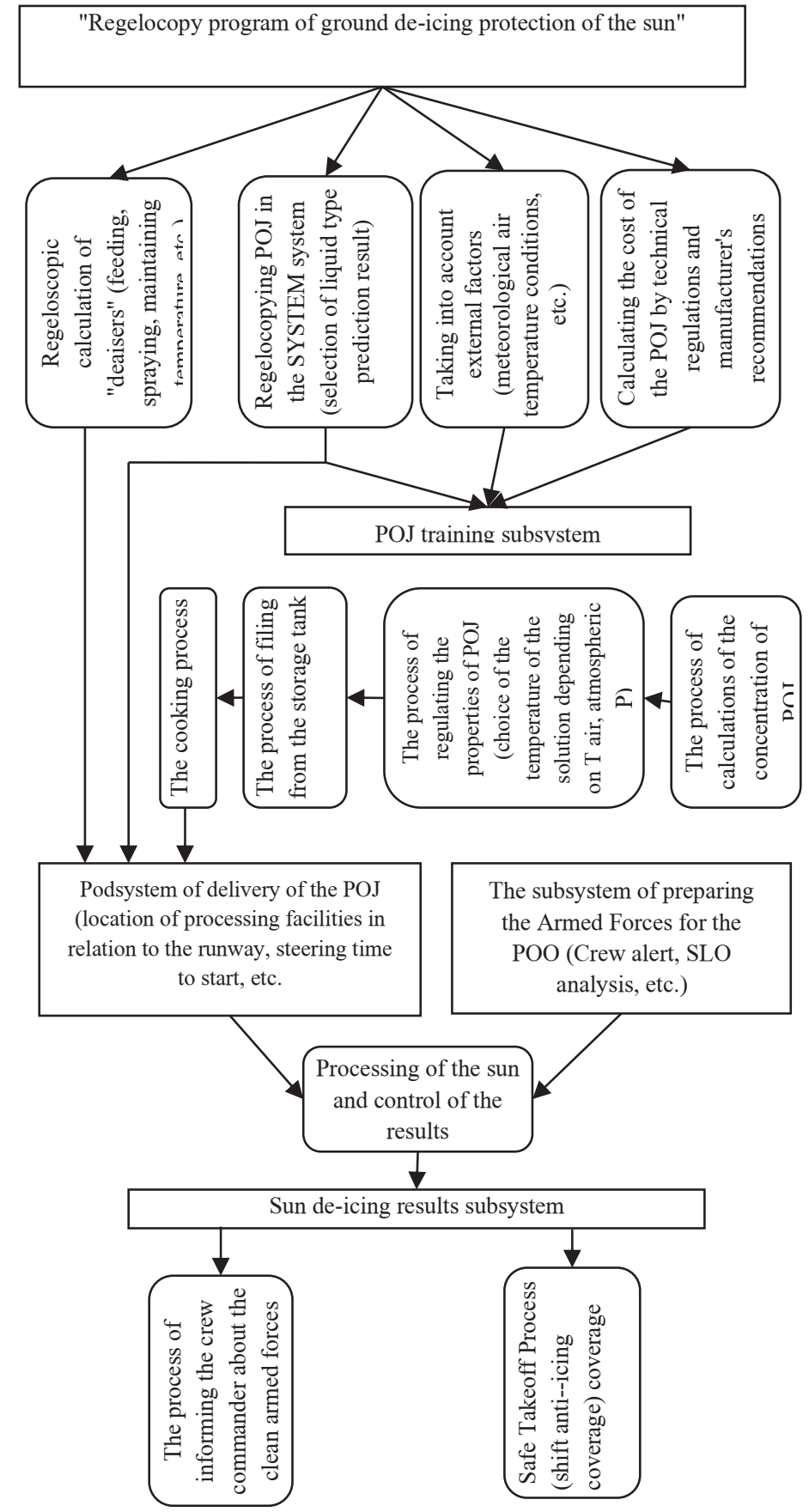

Fig. 2. Regeloscopic program of ground de-icing protection of the sun

This concept, unlike technical guidance, regulations and recommendations for preparation for use and application, will determine not only the regime parameters of the process of preparation and use of POJ to obtain the required indicators, but also the technology of fluid preparation, regulation of its properties, and become the basis for automation of quality management at the POO stage [21]. 


\section{Conclusions}

In this article, we believe that the evolution of "quality control" of individual stages in "quality management" - de-icing fluid as an object to ensure the safe take-off of the sun, all stages of the life cycle and automation of this process - is natural. This will simplify the analysis of the state and control of products, increase economic efficiency and environmental safety, while implementing the task of ground, physical and chemical treatment of the surface of the Armed Forces, the integrated ability to provide effective ground-based treatment of the armed forces, with the preservation of de-icing effect by the formation of a "shifted" protective atyobledine layer on the surface of the Armed Forces, providing accident-free take-off, within the concept of "clean aircraft".

Based on the accumulated knowledge bases about the SITE object, the segment of its life cycle - "The quality of the POJ at the POO stage" has the right to exist within the framework of the automation of the process. "Management of the quality of the POJ."

Introduced the concept of "Regeloscopic program of ground de-icing protection of the armed forces" - allows to introduce information program matic support for the PROCESS process using databases and control systems.

\section{References}

1. SAE AMS 1424. Deicing/Anti-Icing Fluid, Aircraft, SAE Type I

2. SAE AMS 1428. Aircraft Deicing/Anti-Icing Fluid, Non-Newtonian (Pseudoplastic), SAE Types II, III, and IV

3. O. M. Bondarenko, M. V. Makarov, O. Y. Stradomsky, A. Y. Filatov, Control of the quality of de-icing liquids at stages from production to their use in airports, 31

4. GOST P 54264-2010. Air transport. The system of maintenance and repair of aviation equipment. Aircraft de-icing techniques and procedures. General requirements.

5. Y. Deniskin, P. Miroshnichenko, A. Smolyaninov, Geometric modeling of surfaces dependent cross sections in the tasks of spinning and laying, E3S Web of Conferences (2019) DOI: $10.1051 / \mathrm{e} 3$ sconf/201911001057

6. I. Pocebneva, Y. Deniskin, A. Yerokhin, V. Artiukh, V. Vershinin, Simulation of an aerodynamic profile with sections of ad hoc concavity, E3S Web of Conferences, 110, 01074

7. Y. I. Bityukov, Y. I. Deniskin, I. V. Pocebneva, Construction of Smooth Biorthogonal Waves on Triangulated Spaces, Proceedings - 2019 International Russian Automation Conference, RusAutoCon (2019) DOI: 10.1109/RUSAUTOCON.2019.8867785

8. E. Matys, Y. Deniskin, E. Stativa, D. Shlychkov, Special features of obtaining fine powders for additive technologies, E3S Web of Conferences (2019) DOI: 10.1051/e3sconf/201911001057

9. I. M. Artamonov, A. R. Deniskina, Yu. I. Deniskin, Formation of a single information object for targeted solutions in the aerospace industry, Science Industry Defense Proceedings of the XIX All-Russian Scientific and Technical Conference: in 4 volumes. Ed. S.D. Salenko, 57-61 (2018)

10. Y. I. Bityukov, Y. I. Deniskin, Chaikin algorithm and its generalization, 2016 Dynamics of Systems, Mechanisms and Machines, Dynamics, 7818981 (2016)

11. Y. I. Bityukov, Y. I. Deniskin, G. Y. Deniskina, Spline wavelets use for output processes analysis of multi-dimensional non-stationary linear control systems, Journal of Physics: Conference Series, 944(1), 012018 
12. A. V. Smolyaninov, I. V. Pocebneva, L. V. Chernenkaya, Mathematical Model of Asynchronous Motor with Frequency-Cascade Regulation, Proceedings - 2019 International Russian Automation Conference, RusAutoCon, 8867604 (2019)

13. O. Dolgov, S. Bibikov, I. Pocebneva, Elements of the synthesis method for the layout of a front-line aircraft, E3S Web of Conference, 110, 01068 (2019)

14. V. E. Mager, V. E. Belousov, E. N. Desyatirikova, S. A. Ivanov, I. V. Pocebneva, Information processing algorithm at creation of optimum structure of the self-adjusted technical system in quality parameters, Proceedings of 2017 IEEE 2nd International Conference on Control in Technical Systems, CTS 8109503, 114-117 (2017)

15. E. N. Desyatirikova, L. V. Chernenkaya, V. E. Mager, Subsystem for On-line Diagnostics of Cutting Process in Flexible Manufacturing, Proceedings - 2019 International Russian Automation Conference, RusAutoCon, 8867814 (2019)

16. E. N. Desyatirikova, L. V. Chernenkaya, V. E. Mager, Method of calculating a priori reliability of elements of complex system, International Russian Automation Conference, RusAutoCon 8501747 (2018)

17. G. N. Kravchenko, Yu. I. Popov, K. G. Kravchenko, Probability prediction of the appearance of overload cycles of variable load case in parts of the aircraft chassis, IOP Conference Series: Materials Science and Engineering, 734(1), 012014

18. E. Protopopov, S. Dobrykh, Y. Trofimova, P. Malenko, A. Valter, Protopopov A, Reflection of strengthening results in values of generalized degrees of metallicity and covalence is principle to new strategy of designing alloys, Scientific Reports, 10(1), 2050 (2020)

19. A. Dehghani, F. Aslani, The synergistic effects of shape memory alloy, steel, and carbon fibres with polyvinyl alcohol fibres in hybrid strain-hardening cementitious composites, Construction and Building Materials, 252, 119061 (2020)

20. A. A. Smagin, O. S. Dolgov, I. V. Pocebneva, On the Issue of Increasing the Stability and Controllability of Aircraft of Non-Traditional Schemes When Moving on the Ground Proceedings 2020 International Russian Automation Conference, RusAutoCon, 9208082, 920-925 (2020) DOI: 10.1109/RusAutoCon49822.2020.9208082

21. Y. Deniskin, A. Deniskina, I. Pocebneva, S. Revunova, Application of complex information objects in industry management systems E3S Web of Conferences, 164, 10042 DOI: $10.1051 / \mathrm{e} 3$ sconf/202016410042 